\title{
Status of Double Chooz experiment
}

\author{
Emmanuel Chauveau* on behalf of the Double Chooz collaboration \\ Research Center for Neutrino Science \\ E-mail: chauveau@awa.tohokuac.jp
}

Double Chooz is measuring the neutrino mixing angle $\theta_{13}$ via the disappearance of reactor $\bar{v}_{e}$. The experiment relies on the measurement of neutrino spectrum with two identical detectors at different location to the Chooz nuclear power plant : one at $1 \mathrm{~km}$ of reactor cores to observe the disappearance of neutrinos around the first minimum and one around $400 \mathrm{~m}$ to measure the flux before any significant oscillation. Neutrinos are detected by inverse beta decay on free protons in a 8.3 tons liquid scintillator target doped with Gadolinium, leading to coincidence signals of a prompt positron with a delayed neutron capture on Gadolinium and allowing effective background suppression. Double Chooz has been running since 2011 with the far detector only, providing on the scene novel analysis techniques like reactor rate modulation study which gives independent measurement of $\theta_{13}$ and background rate, new efficient background vetos techniques or analysis using neutron capture on Hydrogen with independent data sample. Using far detector only data, the latest Gd result is $\sin ^{2}\left(2 \theta_{13}\right)=0.090_{-0.029}^{+0.032}$ and a new $\mathrm{H}$ analysis is presented which yields to $\sin ^{2}\left(2 \theta_{13}\right)=0.098_{-0.039}^{+0.038}$ and demonstrates the capability of precise measurement of reactor $\bar{v}_{e}$ without Gd loading.

The European Physical Society Conference on High Energy Physics

22-29 July 2015

Vienna, Austria

* Speaker. 


\section{Introduction}

Neutrino oscillations phenomenon between the standard three-flavor framework are described by three mixing angles $\theta_{12}, \theta_{23}$ and $\theta_{13}$, two independent mass-squared differences $\Delta m_{21}^{2} \Delta m_{31}^{2}$ and one $\mathrm{CP}$-violation phase $\delta_{C P}$. The major remaining puzzles of these flavor oscillations are the sign of $\Delta m_{31}^{2}$ known as hierarchy of neutrino masses and the possible existence of a CP-violation. $\theta_{13}$ has been the latest parameter determined to be non-vanishing due to its significant smallness with respect to other mixing angles. Its measurement is opening the way to design future project aiming to probe for $\mathrm{CP}$-violation in the leptonic sector.

\section{Experimental concept}

Double Chooz experiment aims to measure $\theta_{13}$ by observation of the disappearance of electron antineutrino $\bar{v}_{e}$ at its first minimum, where oscillations driven by $\theta_{12}$ and $\Delta m_{21}^{2}$ parameters stay negligible (Fig. 1). It benefits the intense flux of antineutrinos emitted inside nuclear reactors in the pure electronic flavor by $\beta$ decays from fission products. The experimental design is based on two identical detectors located at two different locations : a far detector at a km-scale baseline measuring the spectral-dependent deficit of neutrinos and a near detector to characterise flux and spectrum before any significant oscillations. A direct comparison of the two spectra results into high precision measurement of $\theta_{13}$ thanks to strong suppression of systematic uncertainties on reactor $\bar{v}_{e}$ flux modeling and detection efficiency.

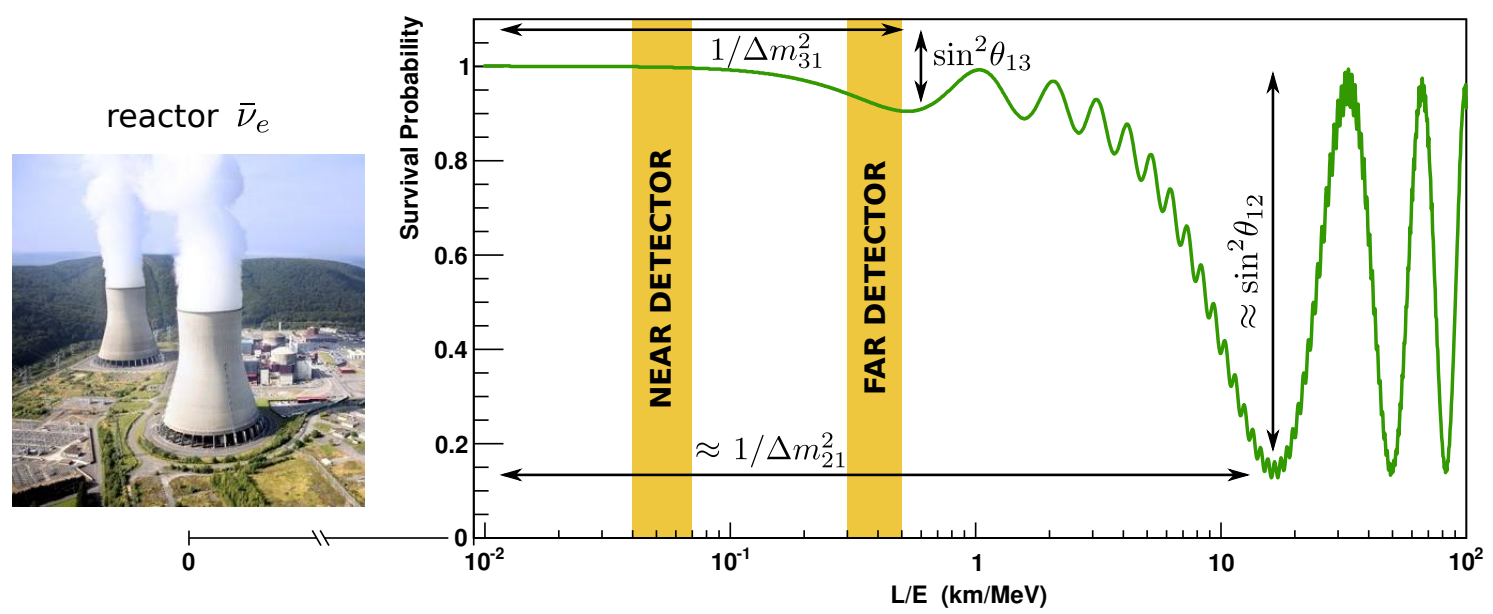

Figure 1: Survival probability of electron antineutrinos as a function of the distance of propagation L over neutrino kinematic energy E showing experimental baselines of the two detectors of Double Chooz.

Electron antineutrinos are detected by inverse beta decay (IBD) on free protons in a large liquid scintillator volume. The reaction generates two particles leading to two signals correlated in time and space. The scintillation and annihilation of the positron gives a prompt signal with a total energy deposit related to the neutrino energy minus $0.78 \mathrm{MeV}$. The delayed signal is generated by the emission gamma ray(s) following the neutron capture on a nucleus. A neutrino target of $10.3 \mathrm{~m}^{2}$ is loaded with $\mathrm{Gd}$, delivering for the delayed signal a large neutron capture cross-section 
and consequently fast capture time $(\sim 30 \mu s)$ with high energy release $(\sim 8 \mathrm{MeV})$. The NT is encircled by a $55 \mathrm{~cm}$ thick Gd-free scintillator volume called gamma catcher (GC), and both NT and GC are surrounded by a $1.05 \mathrm{~m}$ thick transparent mineral oil layer, containing 390 10-inch photomultiplier tubes (PMTs). The delayed signal capture can also happen on $\mathrm{H}$ atoms giving a 2.2 MeV delayed signal and delivering independent data set of IBD with different background level.

\section{Energy reconstruction}

The detector threshold is set around $350 \mathrm{keV}$, far bellow the minimal visible energy of $\bar{v}_{e}$ signal starting from $1.02 \mathrm{MeV}$. For each event, all PMTs waveforms are digitized with $500 \mathrm{MHz}$ sampling on 8 bit ADC. The integrated charge is converted into number of photoelectrons for each PMT using the electronic gain extracted from weekly calibration data with light injection system. The visible energy $E_{v i s}$ is reconstructed based on the total number of photoelectrons $N_{p e}$ on which several correction factors are applied :

$$
E_{v i s}=N_{p e} \times f_{P E / M e V} \times f_{u}(\rho, z) \times f_{s}^{\text {data }}\left(E_{v i s}, t\right) \times f_{n l}^{M C}
$$

where $f_{P E / M e V}$ is the absolute energy scale estimated with ${ }^{252} \mathrm{Cf}$ source at the center of the detector, $f_{u}(\rho, z)$ is the non-uniformity correction of detector response measured using capture of spallation neutron over the full volume and relative to the response at center, $f_{s}^{\text {data }}\left(E_{v i s}, t\right)$ (data only) accounts for time stability correction and $f_{n l}^{M C}$ (MC only) for some charge non-linearity (electronics and readout effects) and light non-linearity (scintillator effects) corrections.

\subsection{Neutrino prediction}

Double Chooz is observing $\bar{v}_{e}$ from the two nuclear reactors of Chooz commercial power plant. The operator Électricité de France is providing complete loading composition of each fuel cycle and operational dynamics of the two reactors including instantaneous thermal power $P^{t h}(t)$ evaluated every minute. From these data, the evolution of fractional fission rates of the four fissile isotopes ${ }^{235} \mathrm{U},{ }^{238} \mathrm{U},{ }^{239} \mathrm{Pu}$ and ${ }^{241} \mathrm{Pu}$ are computed to estimate the mean energy per fission $\left\langle E_{f}\right\rangle(t)$. Reference $\bar{v}_{e}$ spectra are derived from $\beta$ spectrum measured at ILL research facility [1] [2] [3] and FRM-II in case of ${ }^{238} \mathrm{U}$ [4]. The neutrino flux prediction is obtained from :

$$
N_{v}^{e x p}(E, t)=\frac{N_{p} \varepsilon}{4 \pi} \times \sum_{R=1,2} \frac{1}{L_{R}^{2}} \times \frac{P_{R}^{t h}(t)}{\left\langle E_{f}\right\rangle_{R}(t)} \times \sigma_{f}(E, t)
$$

with the number of protons $N_{p}$ in the target, the signal detection efficiency $\varepsilon$, the baseline distance $\mathrm{L}$ to account for solid angle efficiency and the averaged cross-section per fission $\sigma_{f}$. The latter term includes also an absolute normalisation to the flux measurement by Bugey 4 experiment at $15 \mathrm{~m}$ distance [5]. Total systematic error estimated on the flux prediction is $1.7 \%$.

\section{Neutrino selection and background}

In both $\mathrm{Gd}$ and $\mathrm{H}$ analysis, the selection of neutrino candidates is done from a data set of singles energy deposition obtained after applying a muon veto of $1 \mathrm{~ms}(\mathrm{Gd})$ or $1.25 \mathrm{~ms}(\mathrm{H})$ and 


\begin{tabular}{c|c|c} 
& Gd analysis & H analysis \\
\hline Prompt energy & $0.5-20 \mathrm{MeV}$ & $1.0-20 \mathrm{MeV}$ \\
\hline Delayed energy & $4-10 \mathrm{MeV}$ & $1.3-3 \mathrm{MeV}$ \\
\hline Time correlation $\Delta \mathrm{t}$ & $0.5-150 \mu \mathrm{s}$ & $0.5-800 \mu \mathrm{s}$ \\
\hline Spatial correlation $\Delta \mathrm{R}$ & $<1.0 \mathrm{~m}$ & $<1.2 \mathrm{~m}$ \\
\hline Isolation window & {$[-200,+600] \mu \mathrm{s}$} & {$[-800,+900] \mu \mathrm{s}$}
\end{tabular}

Table 1: Selection criteria of neutrino candidates

some criteria on charge isotropy and simultaneity of PMT signals. The selection criteria of prompt and delayed neutrino candidates on energy, isolation, time and space correlation are listed in the table 1.

Three types of background are accounted in the analysis : a) $\beta$-n emitters, mainly ${ }^{9} \mathrm{Li}$, a long live-time cosmogenetic isotope, b) correlated signals due to proton-recoil from spallation fastneutrons and stopping muons decaying into Michel electron, c) accidental background, generated by random coincidence of two singles energy deposits. In the case of $\mathrm{H}$ analysis, this last component is dominant and sizable as the delayed signal is within energy range of natural radioactivity.

Compared to previous Double Chooz publications [6] [7], the selection cut were relaxed to reduce systematic uncertainties on detection efficiency. The resultant increase of background was compensated by the use of new or improved vetos allowing efficient rejection of background with low inefficiency on neutrino signal. In particular, an artificial neural network (ANN) veto was developed to reduce accidental background in $\mathrm{H}$ analysis by exploiting the notable difference between signal and background for 3 variables : delayed energy, space correlation $\Delta \mathrm{R}$ and time correlation $\Delta$ t. As illustrated on the left-hand of Fig. 2, the output of ANN is well controlled and data with accidental background subtracted fully agrees with MC signal. The effectiveness of the veto is demonstrated on the right picture, with a suppression of accidental background by a factor 17 while inefficiency on $\bar{v}_{e}$ signal remains $6 \%$.
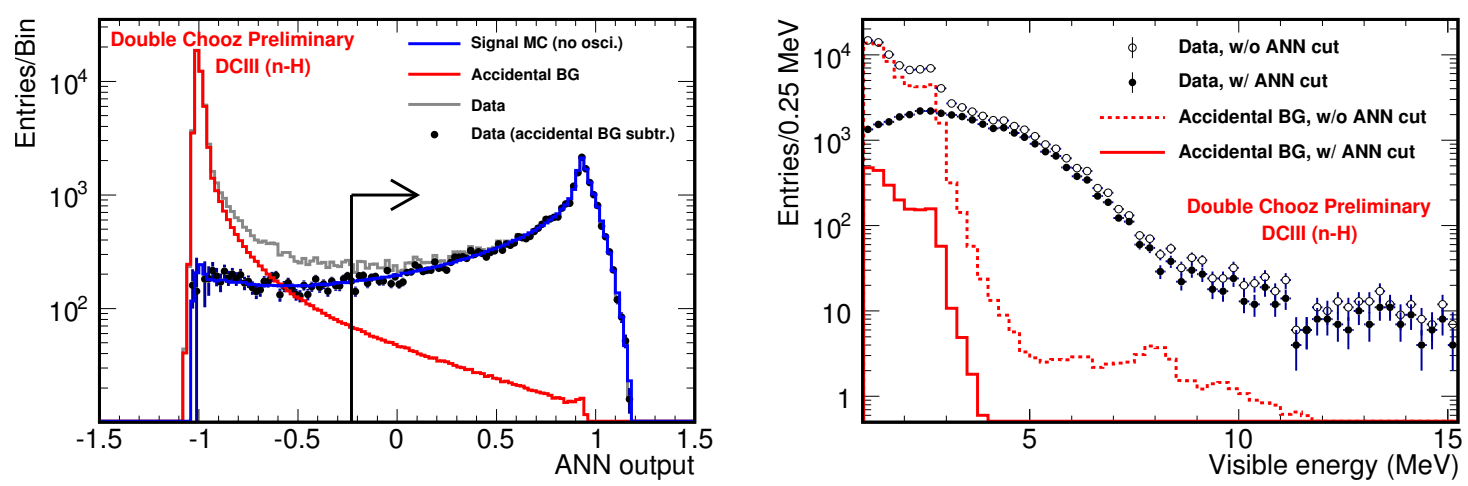

Figure 2: Left: Output of ANN for $\mathrm{H}$ neutrino candidates data (gray), accidental data (blue), signal MC (red) and accidental subtracted data (points). Right: Prompt energy of neutrino candidates (black) and accidental events (red) before and after application of ANN cut $<-0.23$. 


\section{Gd Analysis (2014)}

Over a live-time of 460.67 days, 17351 neutrinos candidates are selected with a total number of background events estimated to $1.64{ }_{-0.17}^{+0.41}$ per day. The oscillation analysis is based on a fit on $\bar{v}_{e}$ spectrum. Data are divided into 40 energy bins with suitable width to analyse both oscillation signature and background rates. Shape of cosmogenetic background comes from ${ }^{9} \mathrm{Li}$ vetoed events, correlated background is assumed to be flat with energy and accidental background is measured using off-time window technique (Fig. 3, left). In the $\chi^{2}$ expression, neutrino candidates are compared to Monte Carlo prediction. A covariance matrix is introduced to propagate systematic and statistical uncertainties in each energy bin and bin-to-bin correlation. Energy scale is allowed to vary by a constraint second-order polynomial function. Reactor-off data is implemented as a pull parameter based on Poisson statistics (rate-only) due to low statistics. Using $\Delta m_{31}^{2}=2.44_{-0.10}^{+0.09} \times 10^{-3} \mathrm{eV}^{2}$ from MINOS [8], the best fit results, illustrated on right Fig. 3, is found at $\sin ^{2}\left(2 \theta_{13}\right)=0.090{ }_{-0.029}^{+0.032}$ with a $\chi^{2}$ of 52.2 for a degree of freedom of 40 [6] excluding non-oscillation hypothesis at $99.9 \%$ confidence level $(3.2 \sigma)$. An unexpected distortion is identified above $4 \mathrm{MeV}$ and is observed to be strongly correlated with reactor power. It does not have significant impact on the result as the fit is mainly constrained at low energy where both statistics and disappearance effects are maximal. Consistent results are obtained with analysis based on rate only analysis and reactor rate modulation (RRM) fit [6].
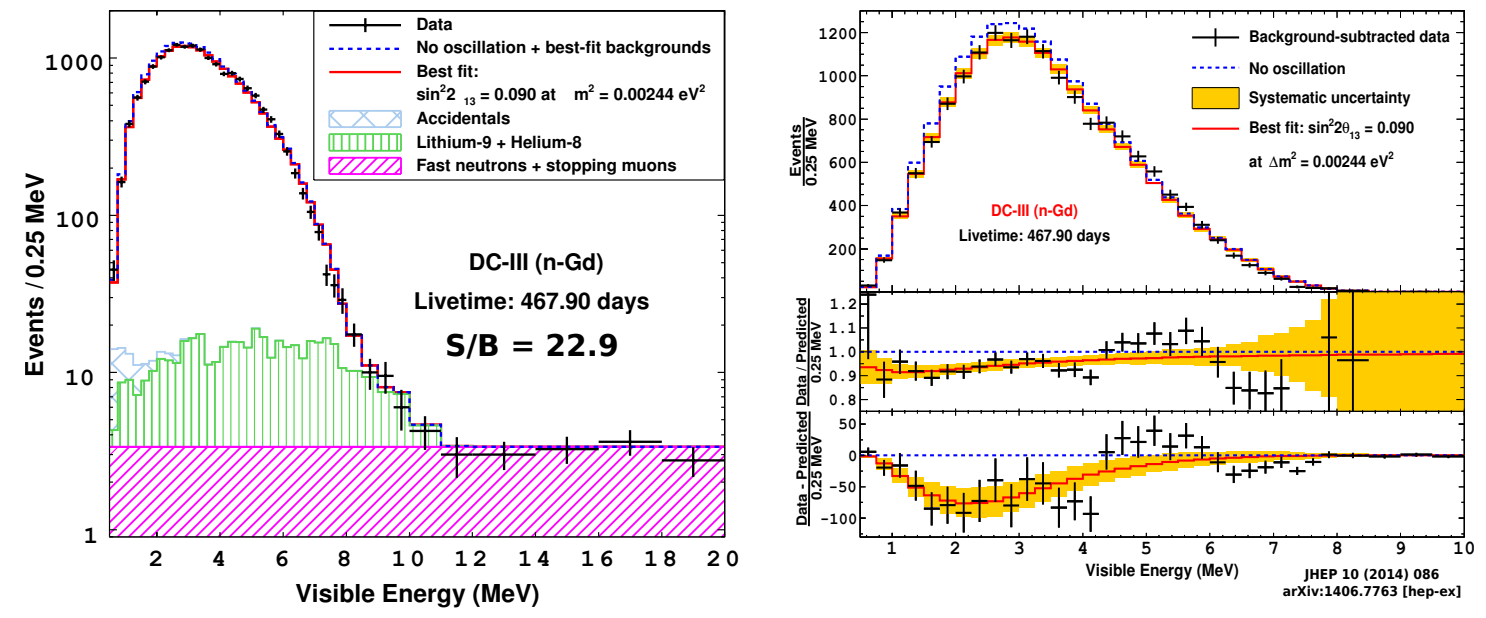

Figure 3: Prompt energy spectrum of Gd neutrino candidates with the three stacked background components and non-oscillated prediction (left) and best oscillation fit results (right).

\section{H Analysis (2015)}

Figure 4 (left) shows the spectrum of $\mathrm{H}$ neutrino candidates together with background components and expected spectrum in the non-oscillation hypothesis. 31835 IBD candidates are obtained during a live-time exposure of 455.57 days with an estimated background rate of $6.83_{-0.36}^{+0.59}$ events per day. Ratio of background-subtracted data to prediction shown on Figure 4 (right) presents a clear spectral-dependent deficit of $\bar{v}_{e}$ due to oscillation. The distortion above $4 \mathrm{MeV}$ reported in 
$\mathrm{Gd}$ analysis is also confirmed in the $\mathrm{H}$ analysis channel, with an independent data set of IBD events in different region of the detector. The rate and shape fit performed on neutrino candidates in $\mathrm{H}$ channels gives $\sin ^{2}\left(2 \theta_{13}\right)=0.124_{-0.039}^{+0.030}$.
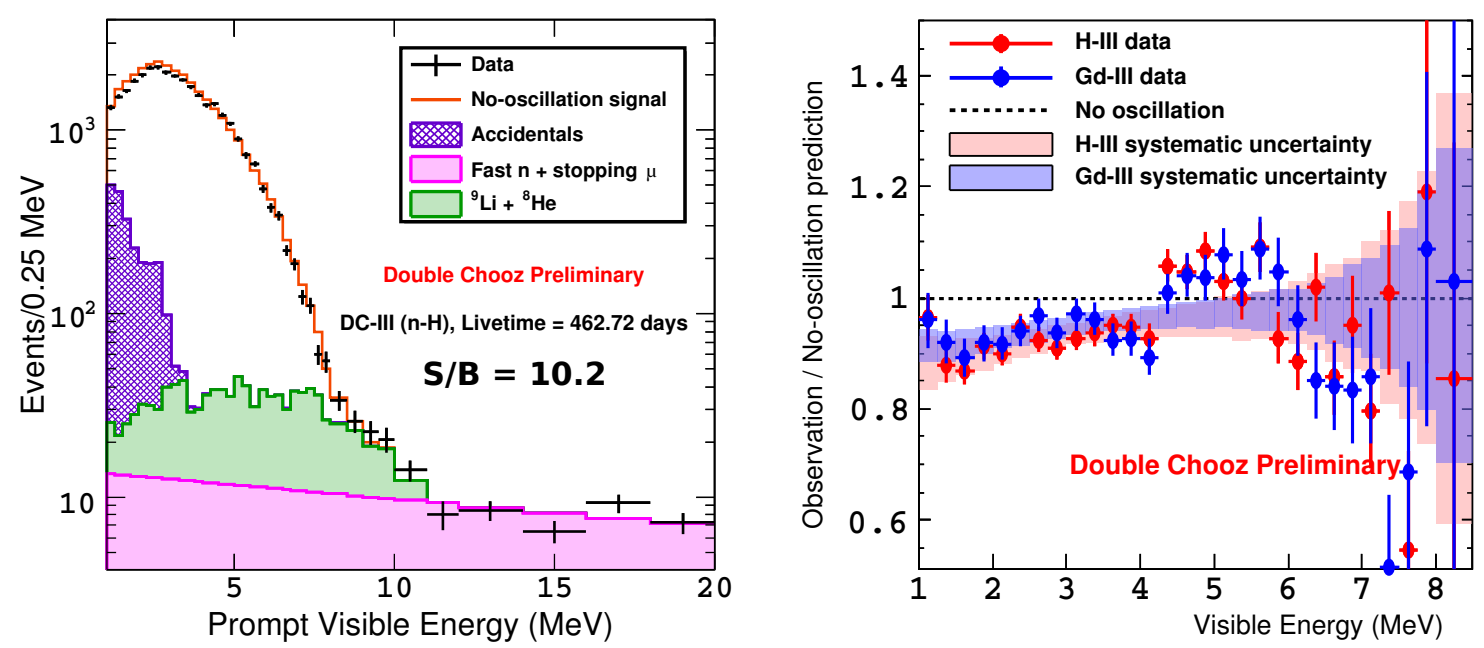

Figure 4: Prompt energy spectrum of $\mathrm{H}$ neutrino candidates with the three background components and nooscillation prediction (left), ratio of background-subtracted data to prediction for $\mathrm{Gd}$ and $\mathrm{H}$ analysis (right).

Observed and expected rate of neutrino candidates are compared for different reactor power conditions. This approach named as RRM analysis gives an independent estimation of $\theta_{13}$ and background rate. The best-fit is obtained for $\sin ^{2}\left(2 \theta_{13}\right)=0.098_{-0.039}^{+0.038}$ and $7.29 \pm 0.49$ background events per day (Fig. 5). Given the observed spectral distortion, this latter RRM analysis is considered as the main $\mathrm{H}$ result. A combined RRM fit for $\mathrm{Gd}$ and $\mathrm{H}$ data sample was performed $\sin ^{2}\left(2 \theta_{13}\right)=0.090 \pm 0.033$. This improves the Gd-only RRM result $\left(\sin ^{2}\left(2 \theta_{13}\right)=0.090_{-0.035}^{+0.034}\right)$ thanks to the increase of statistics by approximately of factor 3 .

\section{Conclusion}

Double Chooz has released updated result on $\sin ^{2}\left(2 \theta_{13}\right)$ using new data-set taken in single detector mode. Several novel techniques of background rejection were worked out for both $\mathrm{Gd}$ and $\mathrm{H}$ analysis. The latest rate and shape fit on Gd data sample gave $\sin ^{2}\left(2 \theta_{13}\right)=0.0900_{-0.029}^{+0.032}$ and an unexpected distortion of the spectrum correlated with reactor power was reported. In the new $\mathrm{H}$ analysis, a predicted signal to background ratio of 10.2 was reached, one order of magnitude larger than in the previous publication [7], especially thanks to the usage of the dedicated ANN veto reducing accidental background by a factor 17 . The RRM analysis of $\mathrm{H}$ neutrino candidates yields a value of $\sin ^{2}\left(2 \theta_{13}\right)=0.098{ }_{-0.039}^{+0.038}$, which demonstrates the capability of precise measurement of reactor $\bar{v}_{e}$ without the usage of Gd loading. The remarkable agreement of all different measurement of $\sin ^{2}\left(2 \theta_{13}\right)$ illustrates the robustness of the analysis and the precision on background knowledge.

Double Chooz has started the two detector phase of data taking early 2015. The current large uncertainties on neutrino flux and detection efficiency will be strongly attenuated bellow $0.1 \%$ level and the experiment expect to reach a final precision of $10 \%$ on $\sin ^{2}\left(2 \theta_{13}\right)$. 

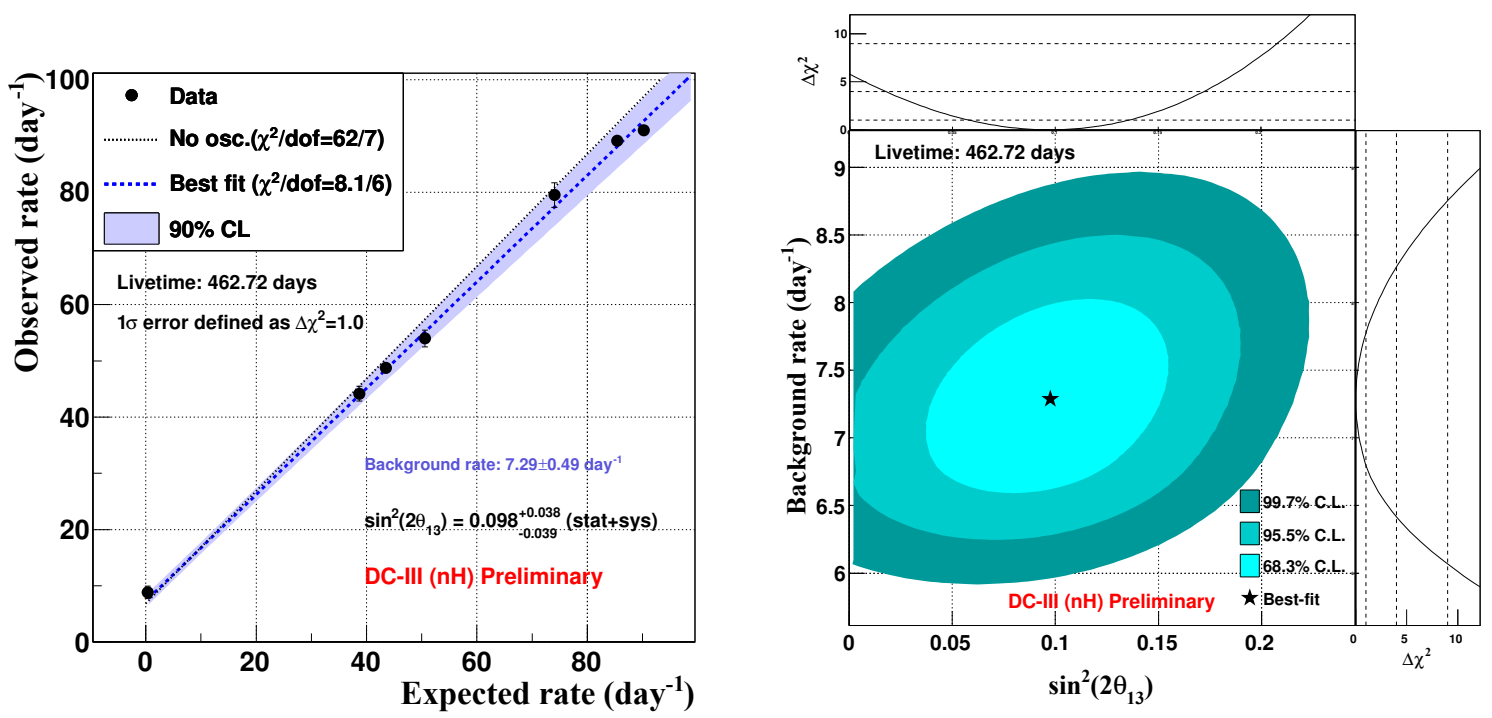

Figure 5: Reactor rate modulation analysis in $\mathrm{H}$ channel: observed rate of neutrino candidates in function of expected rate from reactor flux (left) and best fit results on $\sin ^{2}\left(2 \theta_{13}\right)$ and background rate (right).

\section{References}

[1] Phys. Lett., B160:325-330, 1985.

[2] Phys. Lett., B118:162-166, 1982.

[3] Phys. Lett., B218:365-368, 1989.

[4] N. Haag et al., Phys. Rev. Lett. 112, 122501 (2014).

[5] Y. Declais et al., Phys. Rev. B338:383-389 (1994)

[6] Y. Abe et al., JHEP 10 (2014) 08

[7] Y. Abe at al., Phys. Lett. B723 (2013) 66-70

[8] P. Adamson et al., Phys. Rev. Lett. 112, 191801 (2014) 